\title{
Sound Localization in Adults: Correlation with Varying Pure Tone Averages
}

\author{
Jeong-Sug Kyong ${ }^{1,3}$, Dimode Kim', Mina Park², Myung-Whan Suh³ , Junghak Lee ${ }^{1}$ \\ ${ }^{1}$ Auditory Institute, Department of Audiology and Speech-Language Pathology, Hallym University of Graduate Studies, Seoul, Korea \\ ${ }^{2}$ Department of Otorhinolaryngology-Head and Neck Surgery, Seoul Medical Center, Seoul, Korea \\ ${ }^{3}$ Department of Otorhinolaryngology-Head and Neck Surgery, Seoul National University Hospital, Seoul, Korea
}

\begin{abstract}
Purpose: The present study examined sound localization in terms of varying hearing threshold levels as well as age and sex. Methods: Fifteen healthy volunteers participated with varying mean pure tone averages (mean PTA). Using speaker systems evenly separated by $15^{\circ}$ from $0^{\circ}$ to $75^{\circ}$ to the left and right sides (11 speakers), sound localization error was calculated. Correlation was computed between localization error and various methods of averaging PTA. Three averages were compared: full test frequency range averaging, 6 frequency weighted average used for diagnosing hearing loss and 6 frequency average for Enforcement Decree of Industrial Accident Compensation Insurance Act. Results: First of all, sound localization error positively correlated with age and hearing threshold. The positive correlation was preserved with three averaging methods, with strongest significance with the full range averaging. Conclusion: The present results imply that various averaging methods equally provide significant correlation by the function of localization error.
\end{abstract}

Key Words: Sound localization, Localization error, Pure tone average, Aging.

Received: January 9, 2019 / Revised: January 17, 2019 / Accepted: January 20, 2019

Correspondence: Jeong-Sug Kyong, Audiology Institute, Hallym University of Graduate Studies, 427 Yeoksam-ro, Gangnam-gu, Seoul 06197, Korea Tel: +82-70-8680-5952 / Fax: +82-2-3453-6618 / E-mail: linguis2@hallym.ac.kr

\section{INTRODUCTION}

The ability to locate exact direction and distance of a sound source is critical for surviving, which requires binaural hearing. Localization of a sound depends on several cues, among which time- and intensity-difference constitute major cues. Auditory system computes the difference of the arrival time of a sound at the right and the left ears to determine the direction (interaural time difference). Also, the binaural difference of the intensity of a sound (interaural level difference) is used to determine azimuth (Kerber \& Seeber, 2012; Kwon et al., 2001). In this way, binaural hearing makes the auditory system to find out the differences between the right- and left-sided signals in a very finegrained way and to integrate them into binaural information (Seebacher et al., 2019). In most situations, this occurs automatically and without much effort. For this reason, sound localization error has been examined in groups with various hearing problems, such as in populations with congenital unilateral

(c) This is an Open Access article distributed under the terms of the Creative Commons Attribution Non-Commercial License (https://creativecommons.org/licenses/by-nc/4.0) which permits unrestricted non-commercial use, distribution, and reproduction in any medium, provided the original work is properly cited. hearing loss (Távora-Vieira \& Rajan, 2015) as well as in populations with tinnitus (Hyvärinen et al., 2016) and even in the patients with ischemic cerebral stroke (Przewoźny et al., 2015). Single-sided deaf patients, for example, suffer much in localizing sound sources. To solve this difficulty of localizing due to insufficient binaural hearing, many devices such as, the bone anchored hearing aid, contralateral routing of signal hearing aid, and cochlear implant, were developed and have been widely used (Park et al., 2013; Seebacher et al., 2019).

As such, studies looking at patient group are plenty but normative data are insufficient for now (Dorman et al., 2016). Moreover, the pure tone averages (PTAs) used for examining correlation with sound localization error are various, even though there are sometimes guider consensus papers available (Kerber \& Seeber, 2011), for instance, for unified testing framework in single-sided deafness studies, where PTA is defined as the mean thresholds at pure-tone frequencies of 500,1,000,2000, and 4,000 Hz (Van de Heyning et al., 2016). There is a study which used different PTAs for normal healthy group $(125,250,500,1,000,2,000$, and 4,000 Hz) and for bilateral hearing aided group (250, 500, 1,000, 2,000, and 4,000 Hz) (Dorman et al., 2016), though un- 
derstanding of the specific study and the participants are probable. Averaging of 11 frequencies has also been used $(0.125,0.25$, $0.5,1,2,4,8,9,10,11.2$, and $12.5 \mathrm{kHz}$ ) in a study investigating age-related hearing loss in regard to sound localization (Otte et al., 2013).

In the current study, we aimed to investigate the ability of sound localization in terms of various pure tone averaging methods as well as age and sex. Though PTA has been defined by International Organization for Standardization/1964 and American National Standards Institute/1969 as a mean air-conduction threshold level, several formulae are also in use for clinical or legal purposes (Lim \& Chae, 2012). Therefore, three PTAs were used for comparing in the present study: Averaging full range frequencies tested (Full9), weighted averaging of 6 frequencies used for diagnosing hearing disability (Weight6) and averaging 6 frequencies used for Enforcement Decree of Industrial Accident Compensation Insurance Act (Comp6).

\section{MATERIALS AND METHODS}

\section{Participants}

Fifteen adults participated in the study ( 7 female; 8 male) between the age of 37-72 years. All were right-handed, native Korean speakers and without neurological or neuropsychological history. Before participation, they were screened using Korean version of mini-mental state examination to screen out participants with probable chance of dementia or severe working memory problems (Lee et al., 2002) but all have passed the test and participated the study. Written consent was provided before participation.

\section{Audiological tests}

\section{Pure tone audiometry}

Audiological testing was carried out with the participants sitting in a sound-treated room. Prior to testing, sample stimuli were presented to ensure audibility and to familiarize the listeners with the tasks. Stimuli are pure tones at octave frequencies from $125 \mathrm{~Hz}$ up to $8,000 \mathrm{~Hz}$ including two inter-octave frequencies $0 f$ 3,000 Hz and 6,000 Hz (Audiometer device: Resonance r15c; Resonance, Gazzaniga, Italy)

Full9: PTA by averaging full range frequencies tested: Since we collected from 9 different frequencies, we computed as following.

$$
[(1 \times 125 \mathrm{~Hz})+(1 \times 250 \mathrm{~Hz})+(1 \times 500 \mathrm{~Hz})+(1 \times 1,000
$$
$\mathrm{Hz})+(1 \times 2,000 \mathrm{~Hz})+(1 \times 3,000 \mathrm{~Hz})+(1 \times 4,000 \mathrm{~Hz})+(1 \times$
$6,000 \mathrm{~Hz})+(1 \times 8,000 \mathrm{~Hz})] / 9$

Weight6: PTA by weighted averaging of 6 frequencies were computed as following.

$[(1 \times 500 \mathrm{~Hz})+(2 \times 1,000 \mathrm{~Hz})+(2 \times 2,000 \mathrm{~Hz})+(1 \times$ $4,000 \mathrm{~Hz})] / 6$

Comp6: PTA by averaging 6 frequencies.

$[(1 \times 500 \mathrm{~Hz})+(1 \times 1,000 \mathrm{~Hz})+(1 \times 2,000 \mathrm{~Hz})+(1 \times$ $3,000 \mathrm{~Hz})+(1 \times 4,000 \mathrm{~Hz})+(1 \times 6,000 \mathrm{~Hz})] / 6$

\section{Horizontal sound source localization}

Localization test was also performed in a sound-treated room. Prior to the test, sample stimuli were presented to ensure audibility and to familiarize the listeners with the task. 11 speakers were separated by $15^{\circ}$ from $75^{\circ}$ to the right and from $75^{\circ}$ to the left as shown in Figure 1. Each participant was instructed to identify the speaker of the sound source by reporting the number of the loud speaker and then immediately return to midline waiting for the next sound to be heard. A total of 44 sounds were randomly heard among 11 speakers.

The stimulus used is broadband noise $(125-8,000 \mathrm{~Hz})$ lasting for $500 \mathrm{msec}$ with $10 \mathrm{msec}$ rise and fall each. Intensity of the sound stimuli were set at $65 \mathrm{dBA}$. In the test session, no feedback was provided, to prevent learning of remaining directioncovariant acoustic features (e.g., room acoustics, reflections, and loudspeaker frequency responses). Localization performance was quantified as the total root mean square error of the speaker angles. Therefore, a lower root mean square error indicates better localization skills (Van de Heyning et al., 2016). Percent

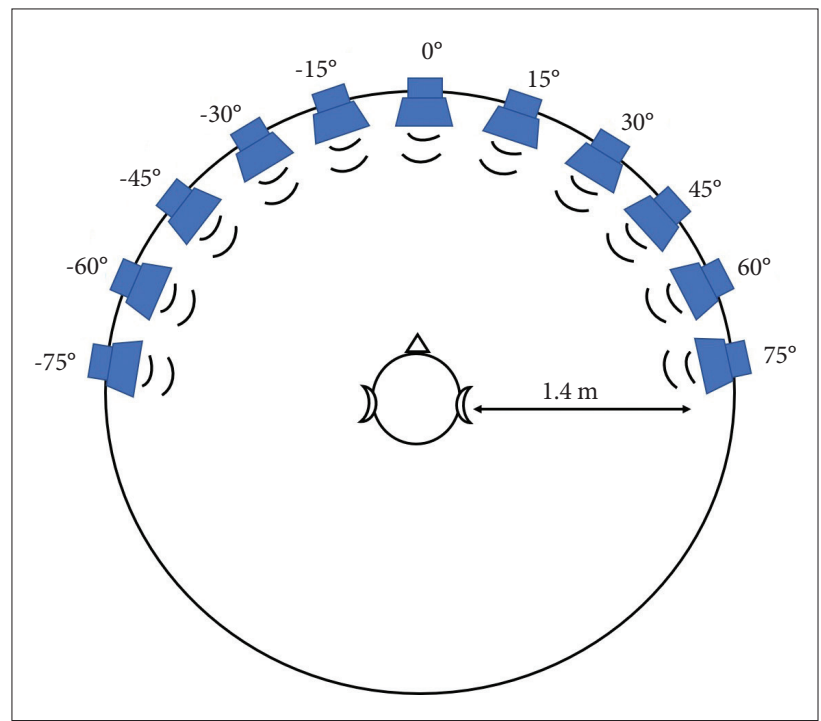

Figure 1. Speaker array for sound localization test. 11 speakers were used from $0^{\circ}$ to $75^{\circ}$ in both to the left and the right equally separated by $15^{\circ}$. Stimulus heard was a $500 \mathrm{msec}$ long broadband noise (125$8,000 \mathrm{~Hz}$ ) with $10 \mathrm{msec}$ rise and fall each. 
correct (\%) was also obtained from each participant.

Simple $t$-test was performed to test group (male and female) difference in sound localization error and correlation was estimated between degree of error and PTAs and age. Computation, estimation and figures were made mainly using Matlab (R2014a; Mathworks, Natick, MA, USA) (Mathworks, 2014a). Data are presented as mean \pm standard deviation (SD) and an outlier was defined as greater than the mean $2 \mathrm{SD}$.

\section{RESULTS}

Mean age was $55.27 \pm 10.77$. Mean degree of error was $5.27^{\circ} \pm$

Table 1. Participant details

\begin{tabular}{cllcc}
\hline $\begin{array}{c}\text { Participant } \\
\text { ID }\end{array}$ & Sex & Age & $\begin{array}{c}\text { PTA* } \\
\text { (dB HL) }\end{array}$ & $\begin{array}{c}\text { Degree } \\
\text { of error }\end{array}$ \\
\hline 2001 & Female & 46 & 5.00 & 5 \\
2002 & Female & 61 & 21.39 & 6 \\
2003 & Female & 56 & 10.28 & 0 \\
2004 & Female & 62 & 15.28 & 8 \\
2005 & Female & 60 & 35.83 & 6 \\
2006 & Male & 72 & 35.56 & 8 \\
2007 & Male & 62 & 21.39 & 5 \\
2008 & Male & 37 & 7.22 & 4 \\
2009 & Male & 56 & 17.50 & 4 \\
2010 & Male & 38 & 8.61 & 2 \\
2011 & Female & 57 & 16.11 & 4 \\
2012 & Male & 59 & 33.89 & 8 \\
2013 & Male & 61 & 32.78 & 13 \\
2014 & Male & 65 & 12.78 & 6 \\
2015 & Female & 37 & 7.50 & 0 \\
Mean & Female 7 & 55.27 & 18.74 & 5.27 \\
$(\mathrm{n}=15)$ & Male 8 & $(\mathrm{SD}: 10.77)$ & $($ SD: 11.00$)$ & $($ SD: 3.33$)$ \\
\hline
\end{tabular}

*Full frequency range was used here. PTA: pure tone average, SD: standard deviation $3.33^{\circ}$ and mean PTA (full range) was $18.74 \pm 11.00 \mathrm{~dB} \mathrm{HL}$ (Table 1). As expected, the degree of error and the percent correct showed negative correlation $(r=0.754, p=0.0046)$. As shown in Figure 2 , age was positively correlated with localization error $(\mathrm{r}=$ $0.604, p=0.017$ ), while men and women did not show any group difference in sound localization error (not-significant, $p$ $=0.1979)$.

\section{PTAs using three different averaging methods}

All three averages and the sound localization resulted in significant positive correlation (Full9, $\mathrm{r}=0.695, p=0.004$; Weight6, $\mathrm{r}=0.609, p=0.016$; Comp6, $\mathrm{r}=0.622, p=0.013$ ) as shown in Figure 3. We also tested with smaller number of samples $(n=13)$ to ensure the sample size for statistical significance (Figure 4).

Since sound localization required both ears, we wanted to know whether asymmetric hearing or hearing level at certain frequencies may affect more to localization. Among the participants, two were asymmetric based on the definition of differences bigger than $15 \mathrm{~dB}$ HL at three contiguous frequencies (Prasad \& Cousins, 2008). Listeners with asymmetric hearing showed larger degrees of error (5.5 degree) than those with symmetric hearing (4.9 degree) but any statistical testing was not possible. Table 2 summarizes the correlation result in the difference of hearing between both ears at 9 frequency ranges and none are significant.

\section{DISCUSSIONS}

This is the first study addressing sound localization in respect to three different pure tone averaging formulae frequently used in Korea. Though guidelines to a unified testing are available in some test areas such as single-sided deafness and tinnitus, various regulations apply various standards. Criteria for the hearing
Figure 2. Age (A) and sex (B) in localizing sound source. All mean data are from both ears. deg: degree, ns: not-significant.
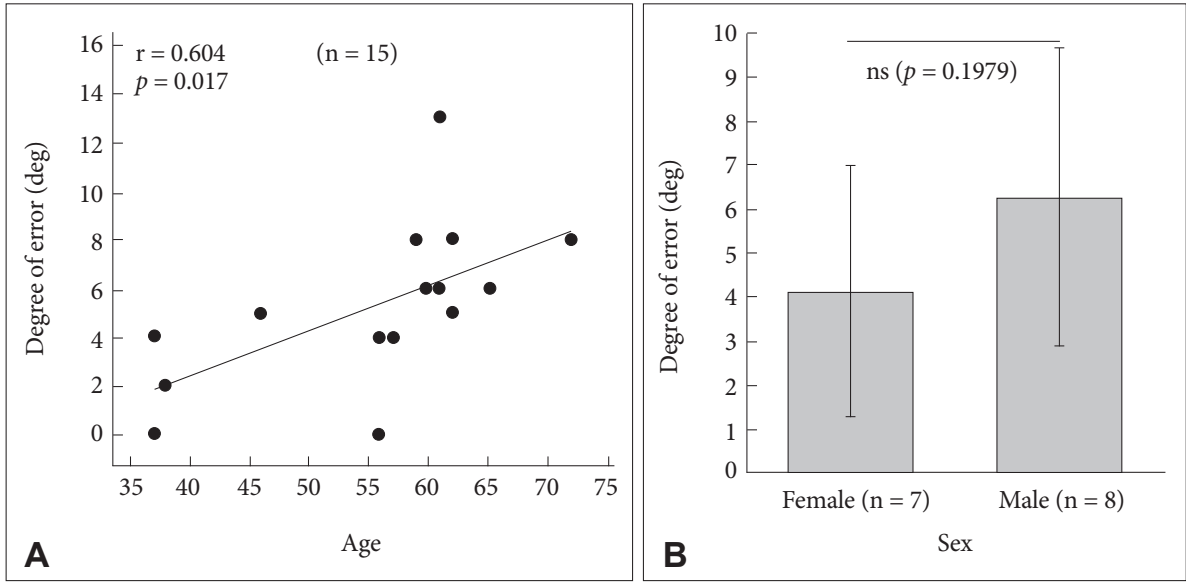

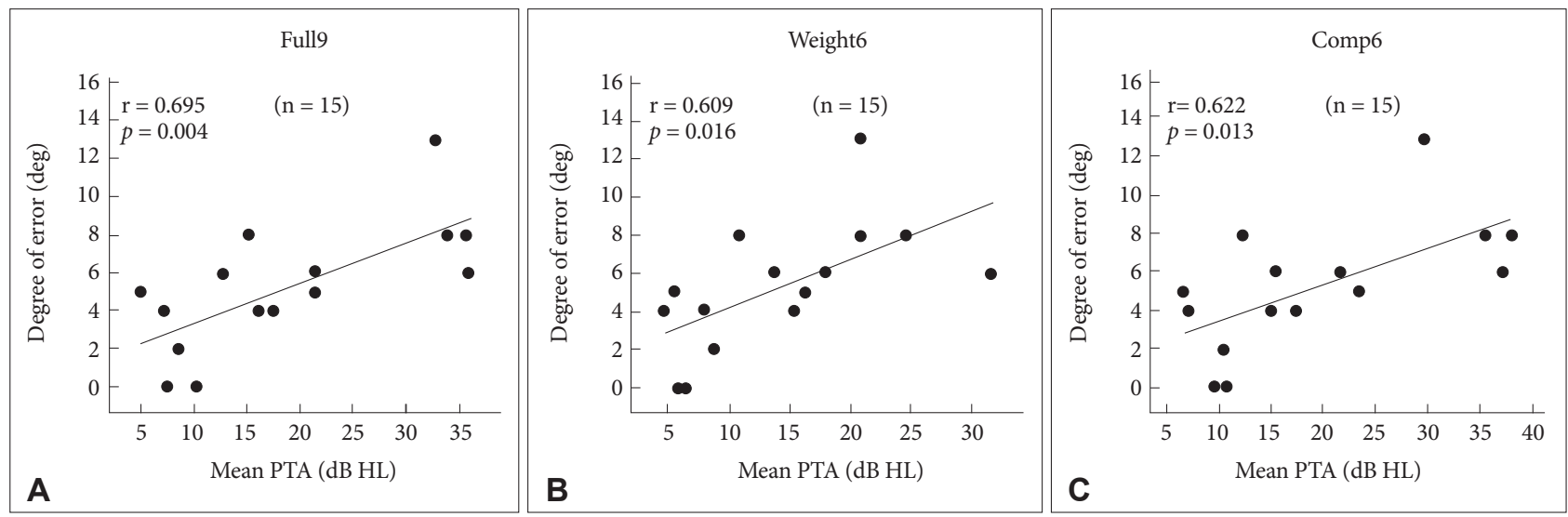

Figure 3. Sound source localization error by the function of mean PTA by full range frequencies (A), by weighted averaging (B), and by averaging 6 frequencies $(\mathrm{C})$ using 15 subject data. All mean data are from both ears. Full9: averaging full range frequencies tested, Weight6: weighted averaging of 6 frequencies used for diagnosing hearing disability, Comp6: averaging 6 frequencies used for Enforcement Decree of Industrial Accident Compensation Insurance Act. PTA: pure tone average, deg: degree of error.
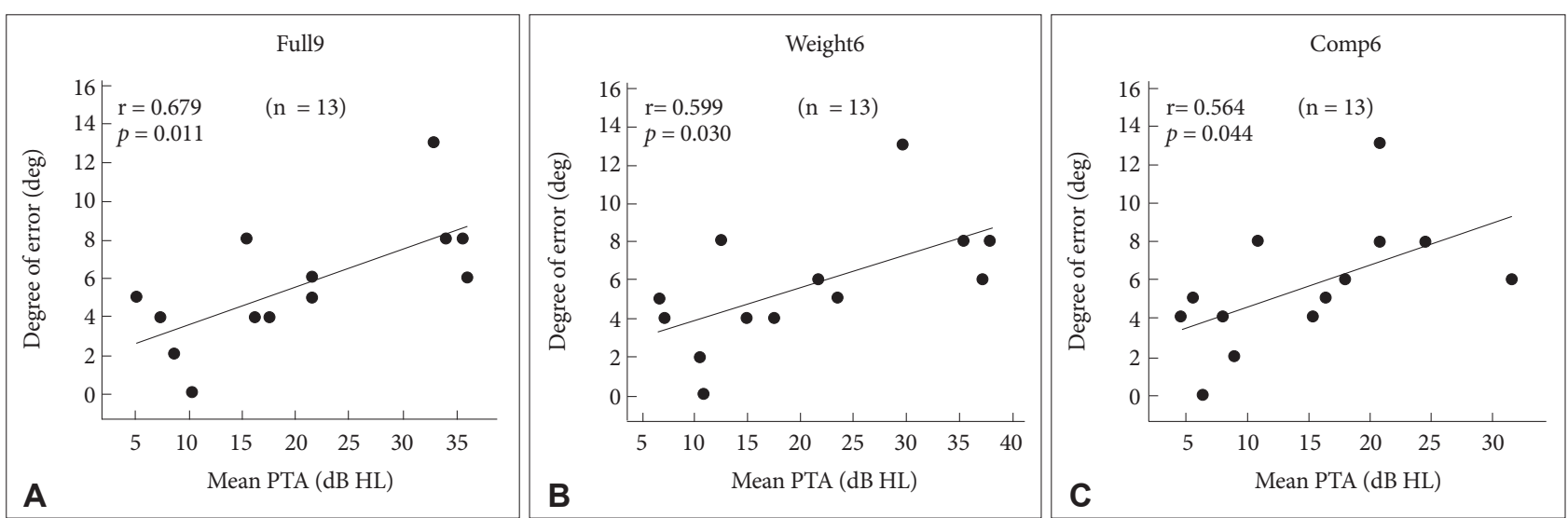

Figure 4. Sound source localization error by the function of PTA by full range frequencies (A), by weighted averaging (B), and by averaging 6 frequencies $(\mathrm{C})$ using 13 subject data. All mean data are from both ears. Full9: averaging full range frequencies tested, Weight6: weighted averaging of 6 frequencies used for diagnosing hearing disability, Comp6: averaging 6 frequencies used for Enforcement Decree of Industrial Accident Compensation Insurance Act. PTA: pure tone average, deg: degree of error.

Table 2. Correlation between sound localization error and difference between both ears at specific frequency in pure tone average*

\begin{tabular}{cccccccccc}
\hline Correlation & $125 \mathrm{~Hz}$ & $250 \mathrm{~Hz}$ & $500 \mathrm{~Hz}$ & $1 \mathrm{kHz}$ & $2 \mathrm{kHz}$ & $3 \mathrm{kHz}$ & $4 \mathrm{kHz}$ & $6 \mathrm{kHz}$ & $8 \mathrm{kHz}$ \\
\hline RHO & 0.1643 & 0.0357 & 0.1241 & -0.0609 & -0.1416 & 0.3173 & 0.4839 & -0.0515 & -0.1627 \\
$p$ & 0.5585 & 0.8995 & 0.6595 & 0.8292 & 0.6148 & 0.2491 & 0.0676 & 0.8553 & 0.5623 \\
\hline
\end{tabular}

*Averaging full range frequencies tested.

impairment grading in Korean disable welfare law are based on the evaluation of hearing impairment and are applied for compensation, indemnification or welfare policy of the disabled. Therefore, three formulae were used in the current study to examine correlations with sound localization error: full frequency range averaging, PTA by weighted averaging of 6 frequencies used for rating disability and PTA by averaging 6 frequencies applying for Enforcement Decree of Industrial Accident Compensation Insurance Act. Despite the small size of the sample, the current study resulted in quite a stable statistical significance. Future study will include the standards for National Pension of
Korea.

As expected, sound localization is affected by hearing level and age. Since various formulae for PTA are in use in the clinical and legal environment, we examined the three different averaging formulae in regard to sound localization in healthy participants. All three methods resulted in reliable significance, with slightly different statistical strengths among three, though. If sound localization error can be used as one of the treatment outcome measures of hearing-aids, cochlear implantation, or other otological problems (Carette et al., 2014; Gifford et al., 2014; Liu et al., 2018), normative distribution along varying PTA is neces- 
sary. Though the sample size is still small, the result of this study may contribute to build normative data for sound localization distribution in various hearing levels within normal range.

Unlike our expectation, the difference of hearing between both ears did not show any statistical significance in localizing sound in the current result. We must admit that this was due to the relatively smaller difference in our participants. With bigger difference, such as in asymmetric hearing or single-sided deafness, we could observe the effect of difference between the ears in locating sound source.

\section{Ethical Statement}

The present study was approved by the Institutional Review Board of Hallym University of Graduate Studies (HUGSAUD148672) and was conducted in compliance with the Declaration of Helsinki, International Conference on Harmonisation Guidelines for Good Clinical Practice.

\section{Acknowledgments}

This research was supported by the National Research Foundation (NRF) of Korea (NRF-2018R1A2B6004788).

\section{Declaration of Conflicting Interests}

There are no conflict interests.

\section{Funding}

This research was supported by the National Research Foundation (NRF) of Korea (NRF-2018R1A2B6004788).

\section{REFERENCES}

Carette, E., Van den Bogaert, T., Laureyns, M., \& Wouters, J. (2014). Leftright and front-back spatial hearing with multiple directional microphone configurations in modern hearing aids. Journal of the American Academy of Audiology, 25(9), 791-803.

Dorman, M. F., Loiselle, L. H., Cook, S. J., Yost, W. A., \& Gifford, R. H. (2016). Sound source localization by normal-hearing listeners, hearingimpaired listeners and cochlear implant listeners. Audiology and Neuro-Otology, 21(3), 127-131.

Gifford, R. H., Dorman, M. F., Sheffield, S. W., Teece, K., \& Olund, A. P. (2014). Availability of binaural cues for bilateral implant recipients and bimodal listeners with and without preserved hearing in the implanted ear. Audiology and Neuro-Otology, 19(1), 57-71.

Hyvärinen, P., Mendonça, C., Santala, O., Pulkki, V., \& Aarnisalo, A. A. (2016). Auditory localization by subjects with unilateral tinnitus. The
Journal of the Acoustical Society of America, 139(5), 2280.

Kerber, S. \& Seeber, B. U. (2011). Towards quantifying cochlear implant localization performance in complex acoustic environments. Cochlear Implants International, 12 Suppl 2, S27-S29.

Kerber, S. \& Seeber, B. U. (2012). Sound localization in noise by normalhearing listeners and cochlear implant users. Ear and Hearing, 33(4), 445-457.

Kwon, K., Park, M., \& Lee, J. (2001). A study of horizontal sound localization ability in normal Koreans. Communication Sciences and Disorders, 5(2), 1-14.

Lee, J. H., Lee, K. U., Lee, D. Y., Kim, K. W., Jhoo, J. H., Kim, J. H., et al. (2002). Development of the Korean version of the Consortium to Establish a Registry for Alzheimer's Disease Assessment Packet (CERAD$\mathrm{K})$ : Clinical and neuropsychological assessment batteries. The Journals of Gerontology, Series B: Psychological Sciences and Social Sciences, 57(1), P47-P53.

Lim, H. W. \& Chae, S. W. (2012). New guides to the evaluation of Korean hearing impairment in 2012. Korean Journal of OtorhinolaryngologyHead and Neck Surgery, 55(3), 144-150.

Liu, Y. W., Cheng, X., Chen, B., Peng, K., Ishiyama, A., \& Fu, Q. J. (2018). Effect of tinnitus and duration of deafness on sound localization and speech recognition in noise in patients with single-sided deafness. Trends in Hearing, 22, 2331216518813802.

Otte, R. J., Agterberg, M. J., Van Wanrooij, M. M., Snik, A. F., \& Van Opstal, A. J. (2013). Age-related hearing loss and ear morphology affect vertical but not horizontal sound-localization performance. JAROJournal of the Association for Research in Otolaryngology, 14(2), 261-273.

Park, M. N., Yoo, S. Y., Chun, Y. M., Moon, I. S., \& Kim, S. H. (2013). Hearing rehabilitation of single-sided deafness: Benefit and selection criteria of bone anchored hearing aid and contralateral routing of signal hearing aid. Korean Journal of Otorhinolaryngology-Head and Neck Surgery, 56(6), 339-345.

Prasad, J. \& Cousins, V. C. (2008). Asymmetrical hearing loss. Australian Family Physician, 37(5), 312-316, 319-320.

Przewoźny, T., Gójska-Grymajło, A., \& Gąsecki, D. (2015). Auditory spatial deficits in the early stage of ischemic cerebral stroke. Journal of Stroke and Cerebrovascular Diseases, 24(8), 1905-1916.

Seebacher, J., Franke-Trieger, A., Weichbold, V., Zorowka, P., \& Stephan, K. (2019). Improved interaural timing of acoustic nerve stimulation affects sound localization in single-sided deaf cochlear implant users. Hearing Research, 371, 19-27.

Távora-Vieira, D. \& Rajan, G. P. (2015). Cochlear implantation in children with congenital and noncongenital unilateral deafness: A case series. Otology and Neurotology, 36(2), 235-239.

Van de Heyning, P., Távora-Vieira, D., Mertens, G., Van Rompaey, V., Rajan, G. P., Müller, J., et al. (2016). Towards a unified testing framework for single-sided deafness studies: A consensus paper. Audiology and Neuro-Otology, 21(6), 391-398. 